Capítulo 8

\title{
Contra el imperialismo mediático: discurso y representaciones etnomediáticas del Movimiento de los Pueblos Indígenas
}

DOI: https://doi.org/10.16921/ciespal.23.28

Vilso Junior Santi e Bryan Chrystian Araújo UFRR

\section{Introducción}

Desde la divulgación de los datos del censo de 2010 realizado por el Instituto Brasileño de Geografía y Estadística (IBGE), Roraima, una Unidad Federativa situada en el extremo norte de Brasil, ha sido reconocida como el estado con el mayor número proporcional de residentes indígenas en el país - alrededor del 11,0\% de la población. En ese momento el número de habitantes del estado ascendía a 450.479 personas. De ellos, 55.922 personas se declararon indígenas, 28.763 hombres $(51,4 \%)$ y 27.159 mujeres (48,6\%).

El importante contingente de auto declarados nativos residentes en el estado nos da valiosas pistas sobre la importancia de la temática indígena y sus movimientos para la región y, en consecuencia, la importancia de promover estudios sobre espacios contra hegemónicos - 
periodísticos, culturales, sociales, políticos y científicos - que contemplen estos sujetos.

En el contexto específico de esta investigación, nos propusimos estudiar cómo se mueven los sistemas de representación en el discurso mediático del portal de internet del Consejo Indígena de Roraima $(\mathrm{CIR})^{1}$, sobre el Movimiento de los Pueblos Indígenas, para analizar cómo el portal del CIR representa los temas relacionados con el Movimiento Indígena. La discusión se basa en las aportaciones teóricas de los Mattelart (1978; 2004), al considerar el campo mediático como un componente esencial en la reproducción de una determinada formación social. Pues, como señalan los autores, los medios de comunicación, como objeto del capital hegemónico, sirven para la conformación de ideas de la clase dominante, contribuyendo a la construcción de una sociedad dependiente (Maldonado, 1999).

Para el caso de este estudio, los determinantes se centran en el posicionamiento de las poblaciones indígenas en el escenario político de Brasil y del estado de Roraima. Las representaciones construidas a partir de esa realidad particular contribuyen a dar concreción a una imagen única de los nativos. Fijan en la memoria colectiva de la población una perspectiva singular sobre la cuestión indígena, en la que los discursos mediáticos configuran no sólo la construcción de un imaginario social por medio de la asimilación de ideas, sino que propagan y efectúan la construcción de sistemas específicos de representación sobre el movimiento indígena (Mattelart, 1978; Maldonado, 1999).

La discusión establecida en esta investigación utiliza una estructura de investigación trasdisciplinar que pretende apoyar el encuentro entre teoría, método y práctica en el desarrollo de los análisis realizados (Mattelart, 1978; Maldonado, 1999). Adoptamos como recorte empírico un corpus de investigación compuesto por 89 publicaciones textualizadas en el portal del CIR - organización con mayor representación institucional del Movimiento de los Pueblos Indígenas en el Estado de

1 Disponible en portugués: https://cir.org.br/site/ Acceso en 13 Abr 2021. 
Roraima. En cuanto al período de análisis, seleccionamos como marco temporal (inicial y final) los meses de abril y diciembre de 2018, período que corresponde a los nueve meses posteriores a la 47 $7^{\text {a }}$ Asamblea General de los Pueblos Indígenas - el evento anual de deliberación política y organizativa más importante para el Movimiento Indígena de Roraima.

La investigación de las cuestiones indígenas, los movimientos sociales, la etnocomunicación, la producción del discurso mediático y las (auto)representaciones indígenas son temas por los que se ha desarrollado gradualmente el interés académico en el contexto local. Sin embargo, sigue presente la percepción de que, a pesar del considerable número de residentes nativos en el estado, los enfoques científicos en el campo de los estudios de comunicación sobre el tema son todavía escasos - siguiendo una tendencia de invisibilización de la causa indígena (y de los pueblos) de Roraima.

Sin embargo, al abordar un movimiento de extrema relevancia cultural y política - el Movimiento de los Pueblos Indígenas - y sus prácticas etnocomunicativas, este estudio demuestra su importancia para los pueblos originarios de la región al rescatar una concepción ampliada de la comunicación, al trabajar con la idea de comunicación en movimiento y al intentar dar visibilidad a formas de comunicación consideradas no convencionales, pero consagradas en estos espacios. También nos dimos cuenta, durante el análisis, de cómo las construcciones históricas, culturales y mediáticas ayudan a perpetuar los discursos y las posiciones políticas y cuál es su injerencia en la estructuración de las representaciones y la definición de sus roles en la colectividad (Mattelart e Dorfman, 1980).

\section{El movimiento de los pueblos indígenas}

Según la perspectiva adoptada por Mattelart (1978) y Maldonado (1999) no es posible entender el presente comunicológico sin ubicarlo previamente en un proceso histórico más amplio, que aclare sus rela- 
ciones con los problemas sociales y filosóficos generales de la contemporaneidad. En este sentido, creemos necesario, antes de abordar las prácticas etnomediáticas del CIR, comprender el proceso histórico de movilización del Movimiento de los Pueblos Indígenas y relacionarlo con un marco teórico que dé concreción e individualidad al movimiento social.

Los pueblos indígenas han reaccionado históricamente a la ocupación de sus territorios tradicionales y se han articulado en defensa de sus culturas. Estos pueblos tienen una historia de resistencia y luchas que se diferencia según la coyuntura social y económica imperante en el país: inicialmente lucharon contra la colonización europea y, posteriormente, contra las oligarquías agrarias brasileñas y sus pretensiones de progreso económico sobre las tierras indígenas.

En sus inicios, las movilizaciones de estos pueblos fueron aisladas y puntuales, limitándose a afrontar los retos inmediatos y a defender comunidades y grupos étnicos concretos. Pero para entender el proceso de movilización del Movimiento de los Pueblos Indígenas, optamos por establecer un contexto histórico común a todas las etnias indígenas del país.

Desde el período de la colonización europea hasta mediados de 1889, con la instauración de la primer República brasileña, los pueblos indígenas sirvieron a los intereses de quienes controlaban el poder: sea como herramientas de mano de obra esclava; como barreras vivas para impedir la penetración de enemigos en las fronteras; como contingentes estratégicos para la ocupación del territorio; o como mano de obra barata para los agricultores y ganaderos invasores (Santos, 2016).

En la primer República brasileña, una nueva postura (aún más capitalista), orientada tanto a la expansión del comercio y la industria como al avance de la economía agrícola, favoreció la aceleración de la ocupación económica de las tierras indígenas y los recurrentes enfrentamientos violentos entre indígenas y no indígenas. En este escenario, diversos sectores de la sociedad civil abogaron por el exterminio total de los pueblos indígenas que, según ellos, impedían el avance de la civilización. 
Este contexto sólo empezó a cambiar en 1908, durante el XVI Congreso de Americanistas en Viena, donde Brasil fue acusado públicamente de masacrar a sus pueblos tradicionales. Como resultado, en 1910 se creó el Servicio de Protección de Indios y Ubicación de Trabajadores Nacionales (SPILTN, acrónimo en portugués) - desde 1918 sólo Servicio de Protección India (SPI) - con el objetivo de resolver el "problema indio" y transformar a los nativos en trabajadores nacionales.

A mediados de la década de 1960, bajo acusaciones de violencia, genocidio e ineficacia, el SPI fue investigado por una Comisión Parlamentaria de Investigación (CPI). El proceso se saldó con el despido de varios funcionarios de todos los niveles, incluidos antiguos directores. Las investigaciones en el SPI coincidieron con la reformulación del Estado brasileño por parte de la Dictadura Militar (posterior a 1964).

Durante este periodo, se reforzó la propuesta de crear un nuevo órgano tutelar responsable por los pueblos tradicionales, con el deber de facilitar su aculturación y acelerar su integración económica. En 1967 se suprimió el Servicio de Protección India (SPI) y en su lugar se creó la Fundación Nacional del Indio (FUNAI), que aun existe hoy.

Paralelamente a la FUNAI, la Iglesia Católica creó en 1972 el Consejo Indigenista Misionero (CIMI), que comenzó a trabajar con los pueblos indígenas en diversas regiones del país, como interlocutor político y representativo de estos pueblos, en sus reivindicaciones por el derecho a la tierra y el reconocimiento de que seguían siendo indios. La Iglesia Católica también es responsable por convocar y organizar las primeras Asambleas Indígenas para discutir las demandas relacionadas con la demarcación de las tierras y la participación efectiva de los sujetos indios en las políticas públicas para los indígenas.

Fueron estas reuniones las que permitieron conocer a los diferentes grupos étnicos del país y reconocer que ellos luchaban por una causa común. Estas acciones fortalecieron sus causas, creando el camino que estructuraría el actual Movimiento Indígena en Brasil. Después de esto, las Asambleas Indígenas se multiplicaron por todo el país, y a finales de los años 1970 y mediados de los 1980, los líderes indígenas 
comenzaron a estructurar nuevas formas organizativas en torno a las entidades indígenas e indigenistas.

La fundación de la Unión de Naciones Indígenas (UNI) en 1979 es el primer resultado de estas articulaciones realizadas durante las reuniones. Una vez creada la primera organización de representación nacional, el Movimiento Indígena se volvió en la consolidación de entidades locales y regionales. En 1984, se creó en Roraima, el Consejo Indígena del Territorio de Roraima (CINTERR), que en agosto de 1990 se convirtió en el actual Consejo Indígena de Roraima (CIR).

La Constitución Federal de Brasil promulgada en 1988, llamada Constitución Ciudadana, también representó una importante victoria para los Movimientos Sociales indígenas. La nueva Constitución estableció el reconocimiento de los pueblos indígenas y el derecho a sus tierras como derechos originarios, anteriores a la creación de los estados y provincias nacionales. Además, la constitución aseguro a los nativos el derecho a seguir siendo indios y a conservar sus grupos étnicos, culturas, lenguas y formas tradicionales de organización, aniquiladas por siglos de contacto interétnico e intentos de aculturación (Brasil, 1988).

Sin embargo, como recuerda Santos (2016), la Constitución de 1988 también es responsable de la creación de la Provincia de Roraima - una medida que promueve un rápido crecimiento demográfico en la región, resultado del intenso flujo de migrantes en busca de la riqueza mineral y de los incentivos gubernamentales para el asentamiento de familias de pequeños agricultores. Esta afluencia contribuyó a desterritorializar a las poblaciones indígenas locales e incitó una serie de conflictos violentos entre los indígenas que luchaban por la protección de su territorio original y los no indígenas que querían ocupar y explotar el territorio de los nativos.

Según Baines (2012) es en este contexto belicoso que los pueblos indígenas de Roraima y del país empiezan a reclamar más respeto por sus culturas y etnias. Es a partir de este proceso de conocimiento y reconocimiento que el Movimiento de los Pueblos Indígena se configura 
como un movimiento étnico, distinto de otros Movimientos Sociales nacionales en Brasil.

\section{El Imperialismo mediático}

Como se ha demostrado en la sección anterior, el Movimiento de los Pueblos Indígenas ha reaccionado históricamente a las formas de opresión y genocidio de la sociedad nacional dominante. En este escenario, los medios de comunicación, como herramienta de poder del capital hegemónico, han servido para la reproducción de esta formación social, ayudando a concretar una imagen única de los pueblos originarios, en la que el discurso mediático propaga y efectúa la construcción de sistemas de representación específicos que responden a los intereses de las clases dominantes (Mattelart, 1978).

En este sentido, los estudios de Mattelart (1976; 1978; 1980; 1997; 2004) aportan contribuciones esenciales para denunciar el control geopolítico de las clases dominantes sobre los medios de comunicación y la información en el mundo, además de evidenciar las condiciones de producción de los medios en una sociedad dependiente. Como explica Maldonado (1999), la preocupación crítica de Mattelart reside en comprender con más detalle las relaciones entre el capitalismo y el poder mediático en las sociedades contemporáneas. Desde este punto de vista, el autor explica que Mattelart está interesado en analizar las más diversas formas en que las clases dominantes y los grupos explotados utilizan los productos mediáticos, los primeros como forma de asegurar el control ideológico sobre las capas menos privilegiadas y los segundos como herramienta de lucha contrahegemónica.

En la concepción de Maldonado (1999; 2002; 2015), el pensamiento de Armand Mattelart viene a situar el análisis de la producción y circulación de la comunicación y la cultura en el contexto de la economía global, destacando las formas en que la ideología opera y crea redes de dominación, con un papel decisivo en la comunicación de masas en América La- 
tina (Mattelart, 1978). Cabe destacar que los trabajos de Mattelart (1978), así como Hall (1997), entienden la ideología como representaciones que actúan legitimando intereses de la clase dominante para la conformación de significados en el espacio social en el que se configura la vida cotidiana.

Trabajando con la Teoría de la Dependencia, Armand Mattelart, Piccini y Michèle Mattelart (1976), abordan los principios organizadores de la ideología (y de las representaciones) en los medios de comunicación en Chile, destacando principalmente los problemas de dependencia informativa de las poblaciones de los países latinoamericanos. Según ellos, las llamadas naciones desarrolladas se aprovecharon de las naciones periféricas de América Latina, sometiéndolas a un proceso de desigualdad y pobreza. Los teóricos critican a los medios de comunicación por reforzar las ideas de un desarrollo dependiente del capitalismo internacional, beneficiando a las clases dominantes que seguían privilegiando este sistema. Según ellos, esta minoría privilegiada, con su poder económico, influye en la producción mediática imponiendo sus propias representaciones y significados sobre el mundo y los sujetos.

Ante esta problemática, una importante reflexión planteada por los Mattelart (2004), en el análisis de los medios de comunicación en su relación con lo social, ha buscado comprender si la sociedad de la información ha logrado compartir de manera más equitativa los diversos recursos mediáticos que permiten el fortalecimiento de la esfera pública de manera abierta y no comercial, o si, por el contrario, ha privatizado el acceso y limitado el compromiso, debilitando, así, el ejercicio de la participación ciudadana en la esfera pública de la organización del sentido.

Al evidenciar que los medios de comunicación están inevitablemente relacionados con el poder económico los Mattelart y Piccini (1976) concluyen que la información es sólo uno de los muchos productos comerciales desarrollados y gestionados por algunos grupos privilegiados. Al aportar esta reflexión, los autores exponen que la clase dominante con su monopolio sobre los medios de producción tiende a 
imponer su particular visión del mundo como algo único. En esta perspectiva, sostienen que el análisis de los medios de comunicación debe descubrir lo que está más allá de la realidad aparente, trabajando en la identificación de los discursos y sus significados inherentes.

Mattelart y Mattelart (1997) también añaden que, en la lectura de las representaciones, los mensajes se revelan llenos de significados, manifestando marcas e intereses de una sociedad. Desde este punto de vista, las representaciones/ideologías se difunden en función de los intereses de un grupo (clase) que está bajo el dominio de los medios de comunicación.

En el libro Para leer al Pato Donald, Armand Mattelart y Ariel Dorfman (1980) también analizan el contenido de los cuentos protagonizados por los personajes de Disney. Los autores observan, a través de la investigación de las narrativas y caracterizaciones de los personajes y ambientes, cómo los productos comunicacionales de la empresa actúan como instrumento de dominación cultural, fijando determinadas representaciones del mundo en la vida social cotidiana.

Una de las principales denuncias destacadas por los autores (1980) se refiere a cómo diferentes personajes que vivían en lugares ficticios que tenían características geográficas y culturales similares a las de México y los países sudamericanos, o incluso a las de África, eran retratados en las historias como vagos, sucios, temerosos y poco inteligentes. Por otro lado, los protagonistas de las historias, identificados como estadounidenses, siempre fueron representados como ejemplos de riqueza, valor, inteligencia y poder.

Otro aspecto muy presente en los relatos es una constante exaltación del capitalismo estadounidense, expresada principalmente a través de la idea de movilidad social, igualdad de oportunidades y democracia absoluta. Otros temas abordados en las historias también tienden a normalizar las posiciones de poder habituales en la sociedad estadounidense. Sin embargo, los ejemplos presentados son suficientes para ilustrar cómo el imperialismo mediático controlado por la clase 
dominante ha actuado para difundir representaciones inexactas de las poblaciones marginadas y sus formas de vida.

Creemos que los medios de comunicación tienen un papel notable en la construcción y estructuración de la realidad y se convierten en objeto de disputa, importando a diferentes campos de la sociedad. En consecuencia, como advierte Maldonado (2002), los medios de comunicación se convierten en fruto vinculante de los conglomerados de comunicación, que usurpan gran parte del espacio mediático para emplear sus matrices de representación, contribuyendo al fortalecimiento de las diversas formas de opresión establecidas en las sociedades capitalistas.

En este contexto, si los medios de comunicación están bajo el control de personas e instituciones privilegiadas, corresponde a las poblaciones marginadas encontrar formas de comunicación contrahegemónicas y alternativas para empezar a negociar significados que actualicen su existencia en el mundo. Estas expresiones alternativas de comunicación surgen como formas de resistencia a las representaciones impuestas por los grandes conglomerados mediáticos - este nos parece el caso de los productos comunicacionales del Consejo Indígena Roraima (CIR)

\section{Etnomedia y los principios de la etnocomunicación indígena}

En las secciones anteriores presentamos el Movimiento de los Pueblos Indígenas y profundizamos en el debate teórico desarrollado por Mattelart (1976; 1978; 1997; 2004) en la comprensión del campo mediático como objeto capitalista, utilizándolo como uno de los componentes centrales en la difusión de representaciones que legitiman las formas de opresión de las clases dominantes.

Ante ello, reflexionamos, según Maldonado (2015), Mattelart y Dorfman (1980), qué formas de comunicación alternativa serían necesarias para subvertir el dominio del capital sobre las poblaciones marginadas. Dicho esto, pretendemos, en este apartado, reflexionar sobre la relación de las comunidades étnicas con la comunicación mediática, junto 
con el papel representativo de los etnomedios indígenas en la construcción de los discursos.

Adoptamos como perspectiva para pensar las prácticas etnomediáticas, las experiencias de los comunicadores indígenas del área, destacando así la relevancia del proceso personal como parte del proceso de construcción del conocimiento (Mattelart y Mattelart, 2004; Maldonado, 2002).

Como hemos visto, Roraima es el estado que proporcionalmente tiene el mayor número de habitantes indígenas autodeclarados del país (IBGE, 2010). Sin embargo, el gran número de residentes indígenas en la región no significa una mayor o mejor cobertura de los medios de comunicación de los temas indígenas. En el día a día de los medios de comunicación, la mayoría de las representaciones de la cultura y la vida cotidiana de las poblaciones indígenas son construcciones idealizadas por individuos y organizaciones inmersas en contextos socioculturales específicos y distintos a los que viven las comunidades.

El ajuste de estas representaciones y su relación con lo representado están, por tanto, sujeta a imprecisiones. Como explica Renata Machado Tupinambá (2016), experta en etnomedia, periodista, productora y cofundadora de Radio Yand $\hat{e}^{2}$, en el periódico online Brasil de Fato ${ }^{3}$ : "La imaginación popular se congeló en la creencia de un individuo conocido con el nombre genérico de 'indio', así definido por el colonizador a partir de los años 1500”.

En esta matriz se evidencia la disputa discursiva y los posibles significados contenidos en los discursos que circulan y que conforman no sólo la construcción de un imaginario social, sino también la construcción de sistemas de representación sobre los Pueblos Indígenas. En este campo de batalla discursivo, es donde surgen y se consolidan las prácticas etnomediáticas indígenas, filosóficamente orientadas, geográficamente localizadas y políticamente útiles (Santi y Araújo, 2019). Estas prácticas comunicacionales híbridas surgen de un proce-

2 Disponible en portugués: https://radioyande.com Acceso en: 13 Abr 2021.

3 Disponible en portugués: https://www.brasildefato.com.br Acceso en: 13 Abr 2021. 
so de mediatización, en el que varios campos de la sociedad, incluyendo aquí los campos étnicos y los movimientos sociales, se apropian de los lenguajes y las lógicas de los medios (Fausto Neto, 2006). Conforme Renata Tupinambá (2016), en un escenario de violencia y disputas por la tierra, la apropiación de las herramientas de comunicación digital ha permitido a los nativos ser sus propios interlocutores.

Aún según Tupinambá (2016) esa apropiación permitió a los indígenas hacer oír su voz y defenderse, demostrando así que la tradición y la modernidad pueden ser aliadas en la preservación de sus culturas y pueblos. A partir de estas consideraciones, la periodista define Etnomedia como "[...] una herramienta de empoderamiento cultural y étnico, a través de la convergencia de diversos medios dentro de una etnovisión. Por eso el uso de este prefijo”, justifica (Tupinambá, 2016).

Denilson Baniwa (2017), también uno de los fundadores de Radio Yandê, en una entrevista para la página de la Revista Usina ${ }^{4}$, preguntado por el concepto de Etnomedia Indígena, respondió que esta es: “[...] como un medio que no se mastica por la masa, sino por el entendimiento de un grupo, de un grupo que sufre las mismas cosas, que ve las mismas cosas y que anhela las mismas cosas".

Las consideraciones de Denilson Baniwa (2017) y Renata Tupinambá (2016) destacan las posibilidades de los pueblos indígenas de buscar su protagonismo negado a través de los medios alternativos, como había aclarado Mattelart (1978), procesos que parten de la apropiación y reapropiación de las lógicas y herramientas del campo mediático. Los etnomedios surgen, así, como una alternativa para la expresión de la identidad étnica y la autonomía política y social de los pueblos indígenas. El uso de esta forma de comunicación por parte del Movimiento de los Pueblos Indígenas de Roraima, entonces, puede caracterizarse como un modo de expresión alternativo-popular que reconfigura las herramientas mediáticas a las necesidades e intereses de su colectividad.

4 Disponible en portugués: https://revistausina.com Acceso en: 13 Abr 2021. 
Además, según Baniwa (2017), el fenómeno relacionado con la aparición de varias plataformas que se centran en la etnicidad en la producción de contenidos de comunicación se denomina "etnocomunicación". El periodista recuerda que en esta construcción cada pueblo establece sus propios formatos para satisfacer sus necesidades.

En la búsqueda de nuevos regímenes de visibilidad, los etnomedios indígenas conjugan prácticas mediáticas, procesos socioculturales y aspiraciones políticas en una configuración comunicacional específica - para proyectar lo indígena más allá de las sociedades anteriores, posteriores o exteriores - provocando malestar. Para ello, según Santi y Araújo (2019), la etnomedia utiliza la etnicidad como componente esencial; la territorialidad como elemento regulador; y el reconocimiento como fin (primero y último).

Allí, tomando los procesos de etnogénesis como guía, los pueblos indígenas se apropian de las herramientas mediáticas para reconstruir y evidenciar su existencia y la de su Movimiento Social étnico, ya sintonizado con este nuevo formato de sociabilidad instituido por el campo mediático. Las herramientas mediáticas étnicas, por lo tanto, y la etnocomunicación indígena resultante, se presentan entonces como la base del proceso de construcción no sólo de una identidad, sino de una matriz de identificación compartida, basada en tradiciones culturales preexistentes o (re)construidas - fundamentales para sostener las acciones colectivas en la actualidad (Bartolomé, 2006).

Según Santi y Araújo (2019), la comunicación practicada por el Movimiento de los Pueblos Indígenas - de orientación filosófica, geográficamente localizada y políticamente útil - establece un nuevo individuo, dispuesto a manifestarse como indio y ser reconocido como tal. Esta comunicación y sus prácticas se mueven en la dirección opuesta a los movimientos de aculturación y tratan de la actualización de las afiliaciones étnicas, a las que sus portadores se habían visto obligados a renunciar a menudo. Se trata, por lo tanto, de los nuevos regímenes de visibilidad política del sujeto indígena y sus movimientos en la sociedad. 
La etnocomunicación y los etnomedios se presentan entonces para los pueblos indígenas como una necesidad y oportunidad de trabajar la comunicación de una manera alternativa, no estereotipada, que represente adecuadamente la cultura, las políticas sociales y la identidad de su pueblo frente a las nuevas formas de organización de la sociedad. Surgen como un mecanismo que puede garantizar el acceso a la comunicación a un grupo históricamente desvinculado del contexto hegemónico de los medios de comunicación convencionales.

\section{Análisis del discurso y preguntas heurísticas}

Utilizamos en la secuencia el Análisis del Discurso (AD) como herramienta metodológica con el fin de comprender el lugar de la cultura contra hegemónica en el proceso de construcción del texto mediático enunciado por el CIR. Pues, según Pêcheux (1988) y Souza (2014), el papel del Análisis del Discurso es destacar el proceso de institución del significado a través del lenguaje y aclarar por qué el significado es uno y no otro.

Es en el análisis del texto mediático - entendido como toda producción material que concibe la interpretación (noticias, fotografías, videos, audios, silencios, etc.) - que buscamos, delimitar las formaciones discursivas y vincularlas a las formaciones ideológicas, para comprender el lugar de decir del sujeto indio y los significados en las producciones textuales que desencadena el CIR (Souza, 2014).

Según el Análisis del Discurso, todo sujeto del lenguaje es ideológico, porque no hay lenguaje sin ideología. Por lo tanto, los significados (creados social e históricamente) surgen de las Formaciones Ideológicas (FI). Una FI reúne un conjunto complejo y múltiple de comportamientos y representaciones que se relacionan con las concepciones en el mundo. Según Souza (2014), la FI representa los significados en su condición primitiva, siendo la ideología su materia prima. Y, como no es posible acceder a la ideología sin un sistema de signos que le dé ma- 
terialidad, esta necesita-se organizarse a través del lenguaje en Formaciones Discursivas (FD).

Las Formaciones Discursivas son, por tanto, manifestaciones de Formaciones Ideológicas en el discurso en una circunstancia singular de enunciación. Benetti y Lago (2007) explican que la FD es una región de sentidos, rodeada por un límite interpretativo que excluye lo que invalidaría el sentido. Al estructurarse mediante FDs, la FI define, mediante un procedimiento de selección llamado Proceso Discursivo, qué sentidos son posibles de ser enunciados y cuáles no. Una vez concluido el proceso discursivo, el sujeto enuncia.

El texto producido a partir del ejercicio enunciativo, la parte visible y material de la lengua, se convierte en la llamada Superficie Lingüística del discurso (Souza, 2014). A partir de esta superficie visible de la lengua es que se puede empezar a conjeturar los significados. En esta investigación, siguiendo los postulados de Souza (2014), tratamos de desandar ese camino a la inversa, buscando en los discursos publicados por el CIR (en su portal de Internet), en las llamadas "marcas textuales" y en lo que quedó fuera de la enunciación, lo qué esos textos pueden significar en el proceso discursivo y cuáles son las características de su discurso. Así podemos identificar qué FD integran y conocer la FI que sustenta sus significados.

Tales marcas textuales funcionaron como puntos de entrada para nuestro análisis y se establecieron a partir de una lectura flotante en la que buscamos las principales características de los textos que debían incluirse o excluirse de nuestro corpus de investigación. Según Souza (2014), el análisis del discurso ay debe estar compuesto por la circunscripción del concepto-análisis, así como por la selección, interpretación y análisis del objeto de estudio a través de las preguntas heurísticas.

Después de la lectura flotante de los materiales para su identificación iniciamos el segundo momento del análisis con la delimitación del concepto-análisis. En nuestra investigación, el concepto-análisis identificado está relacionado con el propio objeto de investigación: el Movimiento de los Pueblos Indígenas. Con ello averiguamos cómo los 
textos de nuestro corpus (entendidos como instrumentos teóricos del discurso) construyeron significados sobre el Movimiento Social.

Con la definición del concepto-análisis, percibimos en el material quién tiene algo que decir sobre el tema, lo que ayuda a identificar en los textos sus principales enunciadores. En este paso, reunimos como corpus de estudio 89 t publicaciones del portal del CIR. De ellos, por saturación y agotamiento de los temas, se seleccionaron dos publicaciones sobre el Movimiento de los Pueblos Indígenas, publicadas entre abril y diciembre de 2018 para un análisis más detallado. A continuación, organizamos las publicaciones a través de Secuencias Discursivas (SD) para destacar las marcas textuales que sustentan las Formaciones Discursivas (FD).

Concluida la delimitación del corpus, iniciamos la actividad de descodificación e interpretación de los textos seleccionados. En este caso, una segunda lectura de los materiales fue esencial para mejorar nuestra percepción sobre los elementos catalogados y sus discursos. Junto con esta segunda lectura analítica, activamos las tres preguntas heurísticas de Souza (2014) - a saber: 1. ¿Cuál es el concepto-análisis presente en el texto? 2. ¿Cómo construye el texto el concepto-análisis? 3. ¿A qué discurso pertenece el concepto-análisis tal y como lo construye el texto? - con la intención de aclarar los significados presentes en los discursos de los textos seleccionados.

Destacamos que la primera pregunta encontró su respuesta en el primer momento del análisis a partir de la definición del concepto-análisis. Con la segunda pregunta, empezamos a buscar un significado para el concepto-análisis durante el propio movimiento de interpretación del texto. Seguimos describiendo y descodificando el corpus hasta saturar este significado, y entonces pasamos a la tercera pregunta. Con ello, identificamos el significado construido en el discurso para aclarar el ejercicio de la ideología en el proceso de textualización (Souza, 2014).

Recordamos que la elección de los textos analizados priorizó dar voz al Consejo Indígena de Roraima, por ser el CIR la mayor representación institucional de los Pueblos Indígenas del estado de Roraima. En vista de ello, circunscribimos el análisis a los nueve meses (de abril a 
diciembre) posteriores a la $47^{\mathrm{a}}$ Asamblea General de los Pueblos Indígenas de Roraima, celebrada en el Centro Cultural Lago Caracaranã, en la Tierra Indígena Raposa Serra do Sol. La Asamblea General de los Pueblos Indígenas reúne anualmente a los pueblos Macuxi, Wapichana, Ingarikó, Patamona, Sapará, Taurepang, Wai-Wai, Yanomami y Yekuana en un amplio programa de debates, análisis económicos y actos culturales. Así, es recurrente encontrar textos sobre las consecuencias de la Asamblea en este periodo de tiempo.

Del material disponible en el portal del CIR, elegimos sólo el contenido mediático textualizado, originalmente escrito y publicado por el CIR, excluyendo así las entrevistas, los artículos de opinión y los textos reeditados. Todos los textos utilizados para el análisis fueron escritos por Mayra Wapichana, una periodista indígena que en ese momento trabajaba como asesora de comunicación del CIR.

Por cuestiones de organización del proceso de análisis, seguiremos las recomendaciones de Benetti y Lago (2007), llamando Secuencia Discursiva (SD) a los fragmentos cortados para el análisis y utilizándolos en las respuestas a las preguntas heurísticas de Souza (2014).

\section{Los resultados y los intentos de respuesta}

Recordamos que las (posibles) respuestas que perseguimos con esta investigación están guiadas por los principios de la Etnocomunicación Indígena, de Baniwa (2012) y Tupinambá (2016); por los preceptos de la Teoría de la Dependencia y la Representación (Mattelart, 1976; 1978; 1997; 2004) y (Hall, 1997) y por los postulados del Análisis del Discurso de Pêcheux (1988) y Souza (2014).

Cuando accionamos la Etnocomunicación Indígena, debemos tener en cuenta que, según Santi y Araújo (2019), ella está marcada por la etnicidad, la territorialidad y la necesidad de reconocimiento. Entonces, utilizando la Teoría de la Dependencia de los Mattelart y Piccini (1976) debemos entender los medios de comunicación son un objeto del 
capitalismo hegemónico, marcado por la construcción y reafirmación de las desigualdades en la sociedad. Ya cuando debatimos la representación y la representación mediática, debemos recordar que, según Hall (1997), se trata de procesos de atribución de significado que fijan, confirman o normalizan determinados estándares, estereotipos o construcciones socioculturales.

Con estos términos, pasamos al análisis del primer texto seleccionado, que fue publicado en el portal del CIR el 12 de abril de 2018, aproximadamente un mes después de la celebración de la $47^{\mathrm{a}}$ Asamblea General de los Pueblos Indígenas de Roraima. El texto lleva el título "Sostenibilidad y buen vivir de los pueblos indígenas de Roraima". Después de la lectura flotante del texto, optamos por describir y explicitar qué sentido del concepto-análisis construye el sujeto.

Utilizamos la pregunta heurística de este texto para identificar las siguientes marcas textuales sintácticas: "fruto del trabajo de los líderes tradicionales" (SD1), "las conquistas y desafíos de los pueblos indígenas" (SD2), "además de enfrentar problemas territoriales" (SD3), "la principal conquista" (SD4), "conquista de las 32 tierras indígenas demarcadas y aprobadas" (SD5), "usufructo exclusivo de los pueblos indígenas" (SD6) ${ }^{5}$.

A través del ejercicio interpretativo, estas marcas textuales nos conducen a un cierto concepto de "Movimiento de los Pueblos Indígenas" percibido, a través de SD1, como parte de un proceso histórico de luchas y conquistas protagonizadas por los líderes tradicionales. Es en SD2, SD3, SD4, SD5 y SD6 que el concepto-análisis "Movimiento de los Pueblos Indígenas" empieza a tomar la forma de un movimiento de lucha por las oportunidades políticas y la garantía de los derechos constitucionales indígenas - esenciales en la organización actual de las comunidades en el estado de Roraima. Estos elementos se ponen de manifiesto cuando el texto habla de los retos, los logros, los problemas

5 Traducción libre de los autores. 
territoriales, las tierras homologadas y demarcadas, así como el usufructo exclusivo de los nativos de la región.

Por último, las marcas seleccionadas piensan en el "Movimiento de los Pueblos Indígenas" como una actividad histórica responsable de garantizar los derechos de los nativos de la región. Como una acción colectiva que debe sobrevivir en manos de nuevos liderazgos indígenas, que necesitan seguir luchando por la garantía de los derechos ya conquistados y por nuevos avances en este ámbito. Estos elementos demuestran claramente cómo los principios de la Etnocomunicación, elaborados por Santi y Araújo (2019) - etnicidad, territorialidad y reconocimiento - actúan de forma recíproca e interdependiente en los productos mediáticos del CIR.

El segundo texto seleccionado fue publicado el 9 de agosto de 2018, Día Internacional de los Pueblos Indígenas. El artículo se titula "VII Marcha de los Pueblos Indígenas de Roraima: dos mil indígenas exigieron el cumplimiento de los derechos garantizados en la Constitución Federal Brasileña de 1988 y el Convenio 169 de la OIT” y es parte del concepto-análisis "Movimiento de los Pueblos Indígenas".

En primer lugar, hacemos una lectura flotante para reafirmar que el concepto-análisis está efectivamente abordado. Luego, mediante la segunda pregunta heurística, nos preguntamos cómo el texto construye el significado del concepto-análisis, es decir, entre las diversas definiciones posibles por la polisemia propia de la lengua, cómo se representa el Movimiento Indígena en el texto.

Algunas marcas llaman la atención: "derechos garantizados en la Constitución Federal Brasileña” (SD9), “amenazas que atentan contra los derechos indígenas" (SD10), "recordar al Estado brasileño sus obligaciones constitucionales" (SD11), "proteger y garantizar derechos indígenas" (SD12), "Recordó las 23 muertes causadas durante el proceso de lucha por la tierra" (SD13), "preocupación por el avance de mineros, madereros, pescadores y otros invasores" (SD14) ${ }^{6}$.

6 Traducción libre de los autores. 
Puestos en relación de paráfrasis, estas marcas construyen un cierto concepto de "Movimiento de los Pueblos Indígenas" que dialoga con los significados encontrados en la primera publicación: una representación marcada por la presencia histórica y la lucha incansable de las poblaciones nativas de Roraima. Incluso se encontraron referencias a las pérdidas ocurridas en las reivindicaciones del movimiento en SD12 y SD13.

Las secuencias reafirman la representación realizada por el CIR como una acción política dirigida a la preservación de los derechos garantizados en las luchas anteriores, así como un recordatorio constante de los sacrificios necesarios para conseguir estos derechos. La calidez de estos logros se pone de relieve para las generaciones futuras que, como las anteriores, deben esforzarse por preservar el legado.

Además de contribuir a los significados expuestos anteriormente, estas marcas discursivas también añaden nuevos significados y contextos al concepto-análisis. A modo de ejemplo, la SDo9 y la SD11 nos recuerdan que las conquistas indígenas son derechos constitucionales. Son marcas textuales que construyen el significado del "Movimiento Indígena" como una articulación de la lucha por un derecho garantizado en la Constitución, teniendo por tanto el mismo nivel de relevancia que otros derechos.

El último y más recurrente de los significados sobre el concepto-análisis presentado en las marcas textuales se encuentra en SD1o y SD14. Estas secuencias discursivas abordan las amenazas recurrentes a las poblaciones nativas que residen en el estado de Roraima. Se trata de problemas que, en la mayoría de los casos, amenazan la integridad de las Tierras Indígenas por la explotación de actividades realizadas ilegalmente en el territorio nativo, como la minería, la madera y la pesca ilegales.

El Movimiento Indígena, en estas situaciones, asume la posición de defensor de su territorio. Es evidente, además de la agencia simultánea de los principios de la Etnocomunicación identificados por Santi y Araújo (2019), los conflictos de representación convocados en los 
textos - que emergen a través de los discursos mediáticos manifiestos, formateados por la representación (mediática) construida por el portal del CIR sobre el tema.

Aquí las herramientas mediáticas también actúan como concreción tecnológica de las representaciones; como puesta en escena de carácter predefinido por Mattelart $(1976 ; 1978)$ y Hall $(1997)$ y como herramientas para transponer perspectivas y patrones representacionales.

A partir de las verificaciones de las marcas textuales (SDs), de la construcción del concepto-análisis de los textos interpretados y de la delineación de la Formación Discursiva principal, podemos finalmente perseguir la Formación Ideológica (FI) desencadenada y las respuestas relativas a la tercera pregunta heurística, para identificar a qué discurso pertenece el objeto discursivo en la forma en que el texto construye (Souza, 2014).

Para ello, optamos por rehacer la tercera pregunta heurística incluyendo algunos elementos específicos relacionados con la temática estudiada. Con este reordenamiento, podemos plantearlo de la siguiente manera: ¿qué discurso representa el Movimiento de los Pueblos Indígenas como acción colectiva para luchar y garantizar los derechos relacionados con la ratificación y demarcación de tierras, la construcción de representaciones políticas, la preservación del medio ambiente, y el reconocimiento y respeto de las identidades étnicas indígenas? Nos damos cuenta de que la respuesta a esta pregunta puede encontrarse en torno a una Formación Ideológica que va más allá de los medios de comunicación y añade el discurso del Indigenato, aunque también tenga la cuestión étnica como discusión subyacente.

Pero ¿cuál sería el discurso del indigenato? ¿Y cuál es su relación con la construcción del concepto-análisis "Movimiento de los Pueblos Indígenas" en los artículos publicados en el portal del CIR? Para encontrar respuestas a estas preguntas, realizamos una breve inmersión histórica para revelar el significado del término, cuyo origen se remonta al Brasil Colonial. 
Según Barbosa (2007), la expresión Indigenato se refiere a una reconfiguración de los territorios indígenas por parte de la ley portuguesa, cuando la Carta Real del 1 de abril de 1680, confirmada por la Ley del 6 de julio de 1775, estableció el principio de que los pueblos indígenas son los dueños originales de sus tierras, quedando siempre reservado su derecho sobre ellas. La argumentación del Derecho del Indigenato deriva del hecho de que los nativos son los primeros habitantes del territorio, constituyendo un derecho congénito. Es decir, hay un derecho originario de los pueblos indígenas sobre su territorio tradicionalmente ocupados antes de la creación del Estado brasileño, por lo tanto ellos tiene prioridad sobre todos los demás títulos de propiedad.

Santos (2016) explica que el Indigenato se hazlo operativo de hecho, a través del concepto de posesión permanente, que no está regulado por el derecho civil y, por lo tanto, es declarativo y una garantía para el futuro. Así, corresponde al Estado proceder al deslinde, tal y como establece el artículo 67 de la Ley de Disposiciones Transitorias de la Carta Magna Brasileña de 1988.

Otro factor importante sobre el discurso del Indigenato es que, según la Constitución Brasileña de 1988, la Tierra Indígena se define por su ocupación y no por demarcación. Así, la Unión debe utilizar parámetros antropológicos para el reconocimiento, como los criterios de organización de una comunidad, sus prácticas, costumbres y etnicidad. La cuestión del reconocimiento étnico entra, de este modo, como base del discurso del Indigenato porque, además de ser utilizada como forma de organización sociopolítica por los grupos para los que es relevante, también es utilizada para la conquista y reivindicación de oportunidades políticas colectivas garantizadas a los grupos étnicos por la legislación (Bartolomé, 2006).

Por lo tanto, el discurso del Indigenato, es lo responsable de determinar las representaciones del "Movimiento de los Pueblos Indígenas" realizadas por el portal del CIR. Ello integra una Formación Ideológica que tiene como principio la circunstancia de ser los indígenas habitantes originarios de la tierra, determinando la transmisión de ese derecho 
a sus descendientes, para su continuidad como pueblos con relaciones étnicas e identitarias precolombinas.

\section{Consideraciones finales}

En la presente investigación, el campo de los medios de comunicación es accionado como el principal difusor de las matrices de representación. La forma en que se realizan estas representaciones contribuye a la organización de los agentes sociales y a la consiguiente actualización/mantenimiento de las posiciones culturales, económicas y políticas impuestas a las distintas colectividades y sujetos (Mattelart, 1976 ; 1978). Es en este contexto que los distintos ámbitos de la sociedad se apropian de las herramientas mediáticas para actualizar los discursos que normalizan determinadas relaciones de poder, privilegiando a unos grupos sobre otros.

Roraima es el estado con el mayor contingente de habitantes indígenas autodeclarados del Brasil (IBGE, 2010). Esta cifra debería demostrar por sí sola la relevancia política y social del Movimiento de los Pueblos Indígenas en la región. Sin embargo, en contra de lo que muchos suponen, su relevancia aún no es amistosa ni está garantizada. La mayoría de las representaciones de las poblaciones nativas son construcciones idealizadas por sujetos que, no exentos de su carga ideológica, reproducen estereotipos de dominación (Benetti y Lago, 2017).

En este estudio, accionamos las prácticas etnomediáticas realizadas por el Portal del Consejo Indígena de Roraima (CIR) como un espacio de lucha y resistencia (Mattelart, 1976; 1978). En este sentido, demostramos en la investigación la esencialidad de pensar en la relación de las comunidades indígenas con la comunicación mediática y el papel representativo de los etnomedios en la construcción de discursos.

Dicho esto, atestiguamos que el portal del CIR se establece en este campo utilizando los principios de la Etnocomunicación Indígena en la búsqueda de nuevos regímenes de visibilidad, combinando aspira- 
ciones ético-filosóficas, geográfico-territoriales y etnopolíticas. En ello, los pueblos indígenas se apropian de las herramientas mediáticas para resaltar la existencia de otros discursos.

Así, a partir del Análisis del Discurso, entendemos que el CIR utiliza las herramientas mediáticas para dar voz y presentar representaciones del "Movimiento de los Pueblos Indígenas" que provienen de los propios sujetos representados a fabricar sus discursos (Mattelart y Mattelart, 1997).

En resumen, lo más importante de esta investigación es la percepción de que, en el escenario del conflicto representacional y discursivo (Mattelart, 1976; 1978), las prácticas etnomediáticas ejecutadas por el CIR, surgen y se fortalecen a partir de un contexto histórico marcado por la lucha y la resistencia, estando localizadas geográficamente y orientadas ideológicamente (Santi y Araújo, 2019).

\section{Referencias}

Baniwa, D. (2017, junho 30). Upurandú resewara: entrevista com Denilson Baniwa. Revista Usina. Disponible en: https:/revistausina.com/artes-visuais/ upurandu-resewara-entrevista-com-denilson-baniwa/

Barbosa, M. A. (2007). Os povos indígenas e as organizações internacionais: instituto do indigenato no direito brasileiro e autodeterminação dos povos indígenas. Revista Eletrônica História em Reflexão, 1(2).

Bartolomé, M. A. (2006). As etnogêneses: velhos atores e novos papéis no cenário cultural e político. Mana, 12(1), 39-68.

Baines, S. G. (2012). O movimento político indígena em Roraima: identidades indígenas e nacionais na fronteira Brasil-Guiana. Caderno CRH, 25(64), 33-44.

Benetti, M., y Lago, C. (2007). Metodologia de pesquisa em jornalismo. Petrópolis: Vozes.

Conselho Indígena de Roraima (CIR). (2018, abril 12). Sustentabilidade e o bem viver dos povos indígenas de Roraima. Recuperado de http://cir.org.br/

Conselho Indígena de Roraima (CIR). (2018, agosto 9). VII Marcha dos Povos Indígenas de Roraima. Recuperado de http://cir.org.br/ 
Brasil. Constituição (1988). Caput do artigo 231 da Constituição. Disponible en http://www.planalto.gov.br/ccivil_o3/constituicao/constituicao.htm

Dorfamn, A., y Mattelart, A. (1980). Para ler o Pato Donald. Rio de Janeiro: Paz e Terra.

Fausto Neto, A. (2006). Midiatização, prática social-prática de sentido. Seminário Internacional da Red Prosul: Mediatizacion y Procesos Sociale. Bogotá: $\mathrm{CNPq} / \mathrm{UNISINOS}$ e Universidad Nacional de Colombia.

Hall, S. (1997). The work of representation. In: Stuart (org) Representation - Culutral representation and cultural signifying practices. Sage/ Open University: London/Thousand Oaks/ New Delhi.

Instituto Brasileiro de Geografia e Estatística (2010). Distribuição da População Indígena. Recuperado de https://indigenas.ibge.gov.br/images/pdf/indigenas/verso_mapa_web.pdf

Maldonado, E. (1999). Teorias críticas da comunicação: o pensamento de Armand. Intexto, (6), 37-60.

Maldonado, E. (2002). A problemática do sujeito e das estruturas: pensar a cidadania a partir do comunicacional: o modelo Mattelartiano. Artigo apresentado no GT Comunicação e Cidadania no XXV Congresso Brasileira de Ciências da Comunicação, Salvador.

Maldonado, E. (2015). Epistemología de la Comunicación: Análisis de la vertiente Mattelart en América Latina (Vol. 1). Ediciones Ciespal.

Mattelart, A., Piccini, M., y Mattelart, M. (1976). Los medios de comunicación de masas; la ideología de la prensa liberal en Chile (No. 04; P92. C47, M3 1976.).

Mattelart, A. (1978). The nature of communications practice in a dependent society. Latin American Perspectives, 5(1), 13-34.

Mattelart, A., y Mattelart, M. (1997). Historia de las teorías de la comunicación. Barcelona. Editora Paidos Comunicación

Mattelart, A., y Mattelart, M. (2004). Pensar as mídias. São Paulo: Edições Loyola.

Pêcheux, M. (1988). Semântica e discurso: uma crítica à afirmação do óbvio. Editora da UNICAMP.

Rádio Yandê (2019). A Rádio. Disponible en https://radioyande.com

Santos, R. M. (2016). Reflexões de lideranças Macuxi e Wapichana sobre as contribuições das TICs para os projetos indígenas locais. Universidade Federal de Roraima (UFRR).

Santi, V. y Araújo, B. (2019). Comunicar para mobilizar: as práticas etnocomunicativas do Conselho Indígena de Roraima. Trabalho apresentado no XXVIII 
Encontro Anual da Compós, Pontifica Universidade Católica do Rio Grande do Sul, Porto Alegre - RS, junho.

Souza, S. (2014). Análise de discurso: procedimentos metodológicos. Manaus: Instituto Census.

Tupinambá, R. (2016, agosto 11). Etnomídia, por uma comunicação dos povos originários. Brasil de Fato. Disponible en https://www.brasildefato.com. br/2016/o8/11/etnomidia-por-uma-comunicacao-dos-povos-originarios. 